\section{Cureus}

Published 06/01/2012

\section{C) Copyright 2012}

Newell et al. This is an open access article distributed under the terms of the Creative Commons Attribution License CC-BY 3.0., which permits unrestricted use, distribution, and reproduction in any medium, provided the original author and source are credited.

\title{
Moyamoya Disease: Current Concepts
}

\author{
David W. Newell ${ }^{1}$, Emun Abdu ${ }^{2}$ \\ 1. Neurosurgery, Seattle Neurosciences Institute 2. Swedish Neuroscience Institute
}

$\square$ Corresponding author: David W. Newell, drnewell@seattleneurosciences.com

Disclosures can be found in Additional Information at the end of the article

\section{Abstract}

Moyamoya disease is a disease of the cerebral vessels, which is characterized by narrowing of the intracranial vessels as they emerge from the base of the skull, and hypertrophy of fine collateral vessels in response to this vessel narrowing. Moyamoya means the puff of smoke in Japanese, and refers to the appearance of the abnormal fine collateral vessels appearing on cerebral angiography in patients with this condition. The most commonly affected vessels are located in the anterior circulation and include the distal intracranial internal carotid artery, proximal middle cerebral artery, and proximal anterior cerebral artery. Moyamoya disease is progressive and the underlying cause is unknown. The stenotic and occlusive process often begins unilaterally, but true moyamoya disease inevitably progresses to involve the cerebral vessels bilaterally.

Patients usually present to medical attention in two distinct age groups. One group of patients presents in childhood and the other group presents in adulthood. There are also two distinct clinical presentations. One group of patients presents with intracranial hemorrhage, most commonly in the basal ganglia or in the ventricles, and sometimes in the subarachnoid space. The other group of patients presents with ischemic symptoms with either transient ischemic attacks or with cerebral infarctions. These infarctions can either be deep perforating artery infarctions, watershed infarctions, or large arterial branch infarctions. Silent infarctions can also be found in some patients at the time of initial presentation, and occasionally patients can present with decreased cognitive function.

The condition was first described in Japan, and much of the published literature describing the condition and what is known about it to date, has been published by Japanese investigators. It has subsequently been recognized that moyamoya disease is a cause of stroke and intracerebral hemorrhage in Western as well as in Asian populations.

Treatment of the condition usually consists of one of two types of revascularization surgery which are termed indirect and direct revascularization. Indirect revascularization involves placing vascularized tissue fed by the external carotid system on to the surface of the brain to allow collateral networks to form and reroute blood flow around the obstructed vessels at the base of the brain. Direct revascularization most commonly utilizes direct microvasular anastomosis between the superficial temporal and the middle cerebral artery (STA-MCA) in moyamoya patients. This paper reviews the current state of knowledge about the disease and also discusses current management strategies.

Categories: Neurosurgery

Keywords: moyamoya, cerebral vessels, stroke, intracerebral hemorrhage, sta mca bypass

\section{Introduction And Background}




\section{Cureus}

Moyamoya disease is a disease of the cerebral vessels, which is characterized by narrowing of the intracranial vessels as they emerge from the base of the skull, and hypertrophy of fine collateral vessels in response to this vessel narrowing. Moyamoya means 'the puff of smoke' in Japanese, and refers to the appearance of the abnormal fine collateral vessels appearing on cerebral angiography in patients with this condition, first described by Suzuki and Takaku [1]. The most commonly affected vessels are located in the anterior circulation and include the distal intracranial internal carotid artery, proximal middle cerebral artery, and proximal anterior cerebral artery. The posterior circulation vessels are less commonly narrowed at early stages; however, they can be involved during the later stages of the disease. Moyamoya disease is progressive and the underlying cause is unknown. The stenotic and occlusive process often begins unilaterally, but true moyamoya disease inevitably progresses to involve the cerebral vessels bilaterally.

Patients usually present to medical attention in two distinct age groups. One group of patients presents in childhood and the other group presents in adulthood. There are also two distinct clinical presentations. One group of patients presents with intracranial hemorrhage, most commonly in the basal ganglia or in the ventricles (Figures 1,2), and sometimes in the subarachnoid space. The other group of patients presents with ischemic symptoms with either transient ischemic attacks or with cerebral infarctions (Figure 3). These infarctions can either be deep perforating artery infarctions, watershed infarctions, or large arterial branch infarctions. Silent infarctions can also be found in some patients at the time of initial presentation, and occasionally patients can present with decreased cognitive function. Occasionally headaches or seizures [2] are the first clinical manifestation which lead to imaging studies. Brain infarctions or hemorrhages most commonly occur in the supratentorial compartment but can also occur rarely in the cerebellum [3].
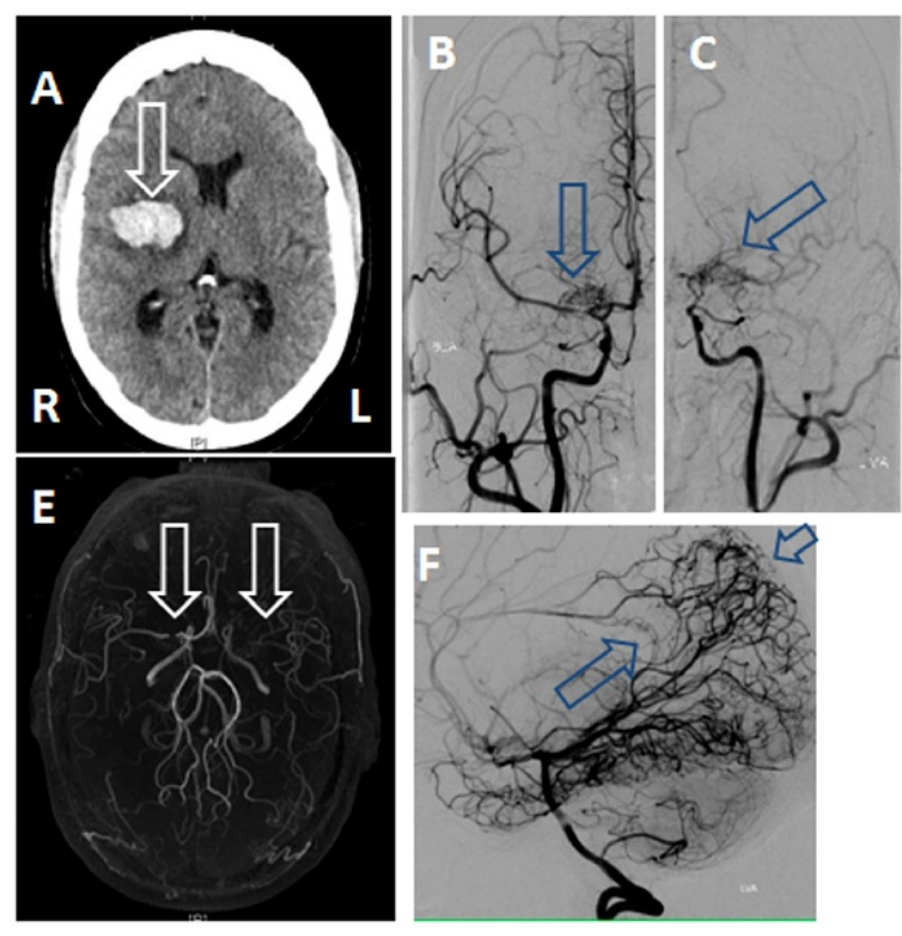
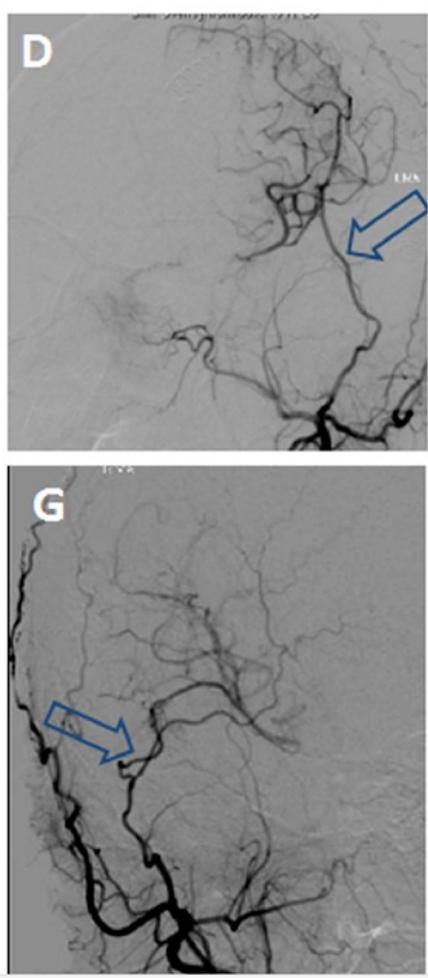

FIGURE 1: This figure illustrates one of the typical presentations and diagnostic findings in an adult patient with hemorrhagic moyamoya disease

This figure illustrates one of the typical presentations and diagnostic findings in an adult patient 


\section{Cureus}

with hemorrhagic moyamoya disease. The patient was found to have an intraparenchmal hemorrhage(arrow) on plain CT (A). MRA (E) showed severe stenosis of the supraclinoid carotid and middle cerebral artery on the right (arrow) and occlusion of the middle cerebral artery on the left (arrow). Cerebral angiography (B right, $\mathrm{C}$ left) revealed classic findings consistent with moyamoya with basal vessel narrowing and collateral vessel changes including bilateral hypertrophy of the fine perforating arteries as well as collateral development from the posterior circulation $(F)$ with extensive leptomeningeal collateral vessels (small arrow) and hypertrophied splenial vessels (large arrow) supplying the anterior cerebral arteries. The patient was treated with bilateral direct superficial temporal to middle cerebral artery bypasses ( $D=$ left, $G=$ right).
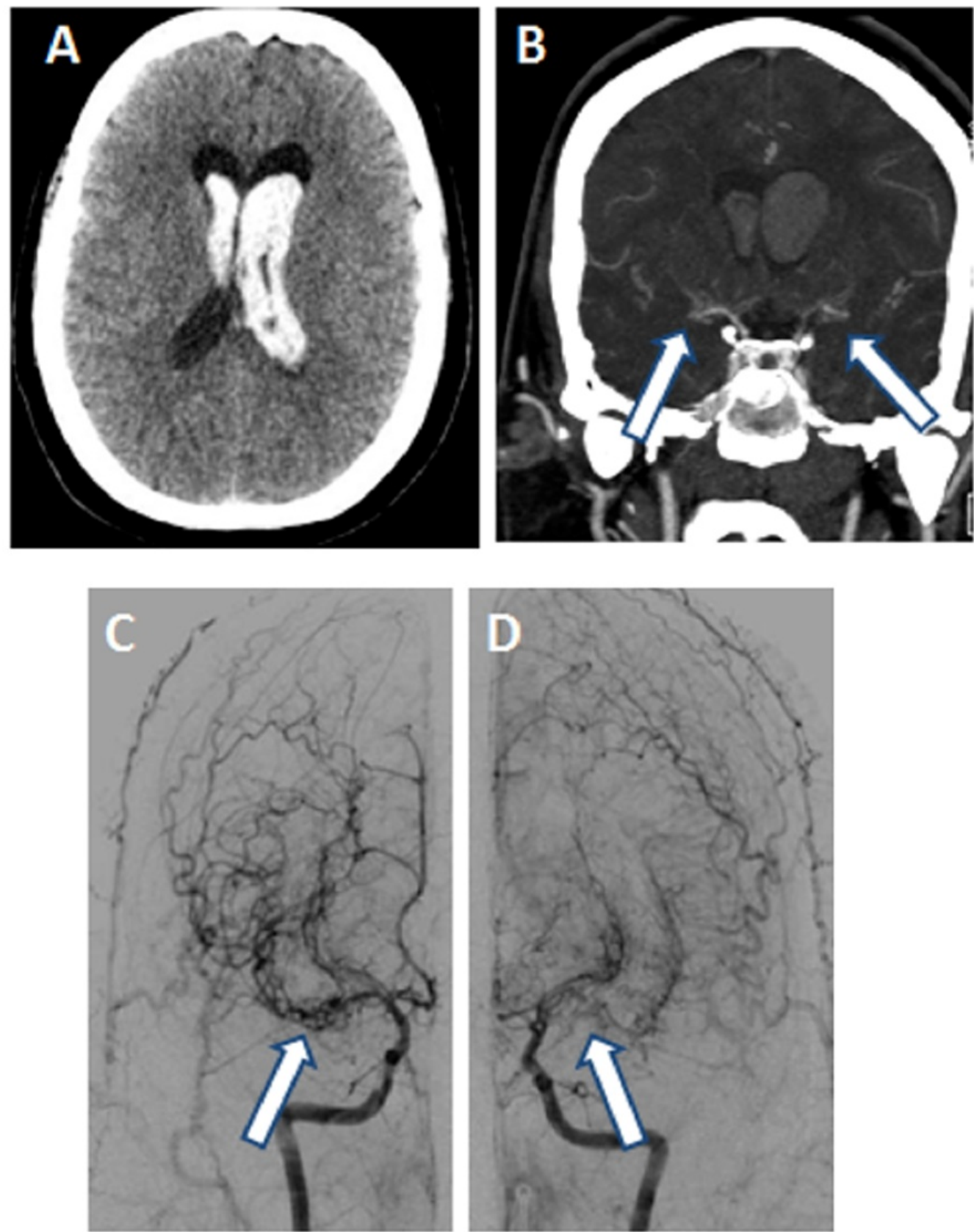

FIGURE 2: Illustration of imaging studies on a patient who presented with acute intraventricular hemorrhage

Illustration of imaging studies on a patient who presented with acute intraventricular hemorrhage (A). Coronal CT angiography (B) suggested the diagnosis of moyamoya which was 
The condition was first described in Japan, and much of the published literature describing the condition and what is known about it to date, has been published by Japanese investigators. It has subsequently been recognized that moyamoya disease is a cause of stroke and intracerebral hemorrhage in Western as well as in Asian populations [4-20].

Treatment of the condition usually consists of one of two types of revascularization surgery which are termed indirect and direct revascularization. Indirect revascularization involves placing vascularized tissue fed by the external carotid system on to the surface of the brain to allow collateral networks to form and reroute blood flow around the obstructed vessels at the base of the brain. Direct revascularization most commonly utilizes direct microvasular anastomosis between the superficial temporal and the middle cerebral artery (STA-MCA) in moyamoya patients. This chapter reviews the current state of knowledge about the disease and also discusses current management strategies.

\section{Review}

\section{Definitions and diagnostic criterion}

Intracranial vessel narrowing has been well recognized in the past as a cause of cerebral ischemia, and can occur from a variety of causes including atherosclerosis, radiation induced arteriopathy, vasculitis, meningitis, sickle cell disease, and vasospasm as well as other more unusual causes. It is therefore important to recognize the difference between moyamoya and other causes of intracranial vessel narrowing. The first description of idiopathic intracranial vessel narrowing which is now known as moyamoya was by Takeuchi and Shimizu in 1957 [21]. The condition subsequently was called moyamoya by Suzuki and Takaku in 1969 [1]. It is well known that the histological changes producing moyamoya are unique and are characterized by an idiopathic non-inflammatory fibrosis [22, 23]. These changes mainly occur in the basal intracranial arteries, however, and these arteries are not appropriate for ante-mortem biopsy as a strategy for confirmation of the disease. The diagnosis of the disease, therefore, is based on a constellation of clinical and imaging findings which can be variable related to the extent and the severity of the findings from the onset of the condition to the late stages of the disease. Due to variations in the extent of changes in some patients, it may be difficult in some cases to make a definitive diagnosis at the time of onset of symptoms if the disease is in the early stages.

When the disease was first recognized in Japan, there were no agreed upon criterion for diagnosis, and other conditions existed which could be confused with moyamoya. A research committee was formed in Japan to publish collected knowledge, establish guidelines and provide information on the condition. This committee was called the Research Committee on Spontaneous Occlusion of the Circle of Willis (Moyamoya Disease) of the Ministry of Health and Welfare of Japan. It was concluded that the diagnosis of moyamoya is based on a collection of clinical and diagnostic imaging features. The guidelines were updated in 1997 [24]. There are no blood or cerebrospinal fluid markers, or other laboratory tests which establish a definitive diagnosis, and routine clinical chemistry and hematological testing is most often normal or changed non-specifically. Due to the fact that moyamoya has a recognized evolution and progression, there were clinical and diagnostic criterion that allowed for a definite diagnosis to be established when all the criterion were fulfilled and a probable diagnosis to be established if some features were missing or had not yet appeared in the progression of the disease. To be characterized as a definite case of moyamoya disease, a patient must have bilateral lesions of 


\section{Cureus}

the cerebral vasculature characterized by narrowing of the supraclinoid carotid arteries and hypertrophied fine collateral vessels on angiography, and they must have an unknown etiology for the vascular lesions, i.e. there must be an absence of other conditions known to cause vascular lesions, including previous radiation treatment, atherosclerosis, autoimmune disease, meningitis, brain tumor, trauma, and other causes. The term moyamoya syndrome has been used to describe patients who have some of the changes of moyamoya disease but do not fulfill the full criterion for the disease (See Table 1 for a summary of diagnostic criterion). There are also criterion to establish a pathological diagnosis on autopsy in patients who died but did not undergo cerebral angiography [24]. 


\section{Cureus}

occasionally present

Clinical symptoms variable

Manner of progression

variable

Can be asymptomatic

Deficits can be transient or persistent

Neurologic deficits can be

mild to severe
Familial occurrence is

Stenosis or occlusion at the terminal portions of the intracranial internal carotid arteries,

proximal portion of the anterior cerebral arteries,

and middle cerebral arteries.

Abnormal vascular networks (moyamoya) are observed in the vicinity of the occlusive (or stenotic) lesions in the arterial phase.

Bilaterality of above findings

Cerebral ischemia is frequently observed in the children, and intracranial hemorrhage in the adults

Childhood form characterized by; hemiparesis, monoparesis, sensory disturbance, involuntary movement, headache, seizures, mental retardation or persistent neurologic deficits hemorrhage is rare atherosclerosis,

autoimmune

disorders,

meningitis,

brain tumor,

Down's

syndrome,

von

Recklinghausen disease,

head trauma,

post-irradiation state, and so forth.)

Adult form characterized by; motor, sensory phenomenon, headaches and seizures similar to those observed in the children. Most have sudden onset of intraventricular, subarachnoid, or intracerebral hemorrhage recovery is variable, some cases may be fatal.

Diagnostic Criterion

Definite diagnosis -

Consider clinical

- cases which fulfill findings listed in II, and III

descriptions in I

- children who have stenosis or occlusion and abnormal vascular networks described in II, and have only a stenosis on the contralateral side

Probable diagnosis

- cases which fulfill all the criterion of II and III except that they are not bilateral

\section{TABLE 1: Clinical Diagnostic Guidelines for Moyamoya Disease according to} Research Committee on Spontaneous Occlusion of the Circle of Willis (Moyamoya Disease) of the Ministry of Health and Welfare of Japan

*Adapted from Fuki

\section{Epidemiology}

The prevalence of moyamoya disease has been noted to be highest in Asian countries, in particular, in Japan and Korea versus Western countries [25, 26]. The prevalence of moyamoya 
disease in Japan has been estimated at three per hundred thousand people, and the annual incidence has been estimated at 0.35 per hundred thousand people. There is a female to male our predominance of 1.8 /1.0. There is also a bimodal distribution of the age of onset of the disease. One peak occurs at approximately age five (pediatric form of the disease) and the other peak of symptom onset occurs at approximately age 40 (adult form). It has been noted that the hemorrhagic presentation of moyamoya has been more common in Asian populations in the adult form, and that the ischemic presentation is more frequently noted in the pediatric form of the disease. It is not clear that this same distribution exists in Western populations, and in fact, a number of Western centers have reported series of adult and pediatric patients where ischemic presentations are more common [9, 27-29]. Uchino, et al. described findings in a collection of 298 patients with moyamoya on the West Coast of the United States in California and Washington between 1987 and 1998. They found the incidence of moyamoya to be .086 per hundred thousand, which appears to be lower than the incidence in Japan; however, this group of patients was multi-ethnic in origin. They found that the incidence was higher in patients of Asian descent $(0.28 / 100,000)$ in this subgroup than in patients who were white $(0.06 / 100,000)$, Hispanic $(0.03 / 100,000)$ or African American $(0.13 / 100,000)$. There have also been reports of moyamoya in Europe with an incidence which is lower than in Japan and similar to that for whites in the United States [7, 8, 30]. With increased availability of improved imaging including more sensitive magnetic resonance (MR), magnetic resonance angiography (MRA), as well as greater resolution of computed tomography angiography (CTA) and more widespread use of transcranial Doppler ultrasound [31], moyamoya disease has been increasingly recognized in Western populations as a cause of stroke in young women.

\section{Genetic aspects}

A number of studies have been performed to attempt to determine the role of genetics in the pathogenesis of moyamoya [32]. Although the exact role of genetics has not been established, the available observations indicate that genetic susceptibility appears to play a major role, and environmental factors may trigger the onset of the disease in susceptible patients. It is known that most cases are sporadic with a higher incidence in genetically susceptible populations. Familial histories of moyamoya have been reported in Japan to be present in $10-15 \%$ of patients, and 13 pairs of twins with the disease have been reported [32-33]. Identical twins have also been described where only one of the twins has developed the disease [34]. It has recently been suggested that an autosomal dominant form with incomplete penetrance exists and that this particular form of the disease maps to chromosome 17q25.3 [35]. Available evidence appears to indicate that a number of genetic mechanisms, including direct autosomal dominant transmission with incomplete penetrance, and also polygenic mechanisms can render individuals susceptible and that environmental factors also play a role in inducing the arteriopathy that is the basis for moyamoya [4, 36-44].

\section{Pathology and pathophysiology of vascular changes}

There are a number of pathological findings reported in moyamoya disease. It is widely believed that the primary pathology is induction of narrowing in the basal intracranial arteries [21], and secondary pathology is found in a number of other sites as a reactive change in the remaining vasculature in response to progressive ischemia of the brain. The overgrowth of the network of fine of vessels emanating from the basal arteries penetrating into the basal ganglia or moyamoya is the change which produces the characteristic blush seen on angiography (Figures 1-3). Histopathological findings in the large intracranial vessels in patients with moyamoya have included severe narrowing of the carotid termination and proximal middle and anterior cerebral artery with fibrocellular thickening of the intima and contraction, folding and duplication of the internal elastic lamina (Figure 4). There is also marked shrinkage and degeneration of the media, sometimes including lipid deposition and ultimately progression to occlusion of the artery lumen [22]. It has been noted that there is an absence of inflammatory 


\section{Cureus}

changes and an absence of atherosclerosis associated with these fibrotic changes which occur in the vessel wall [45].
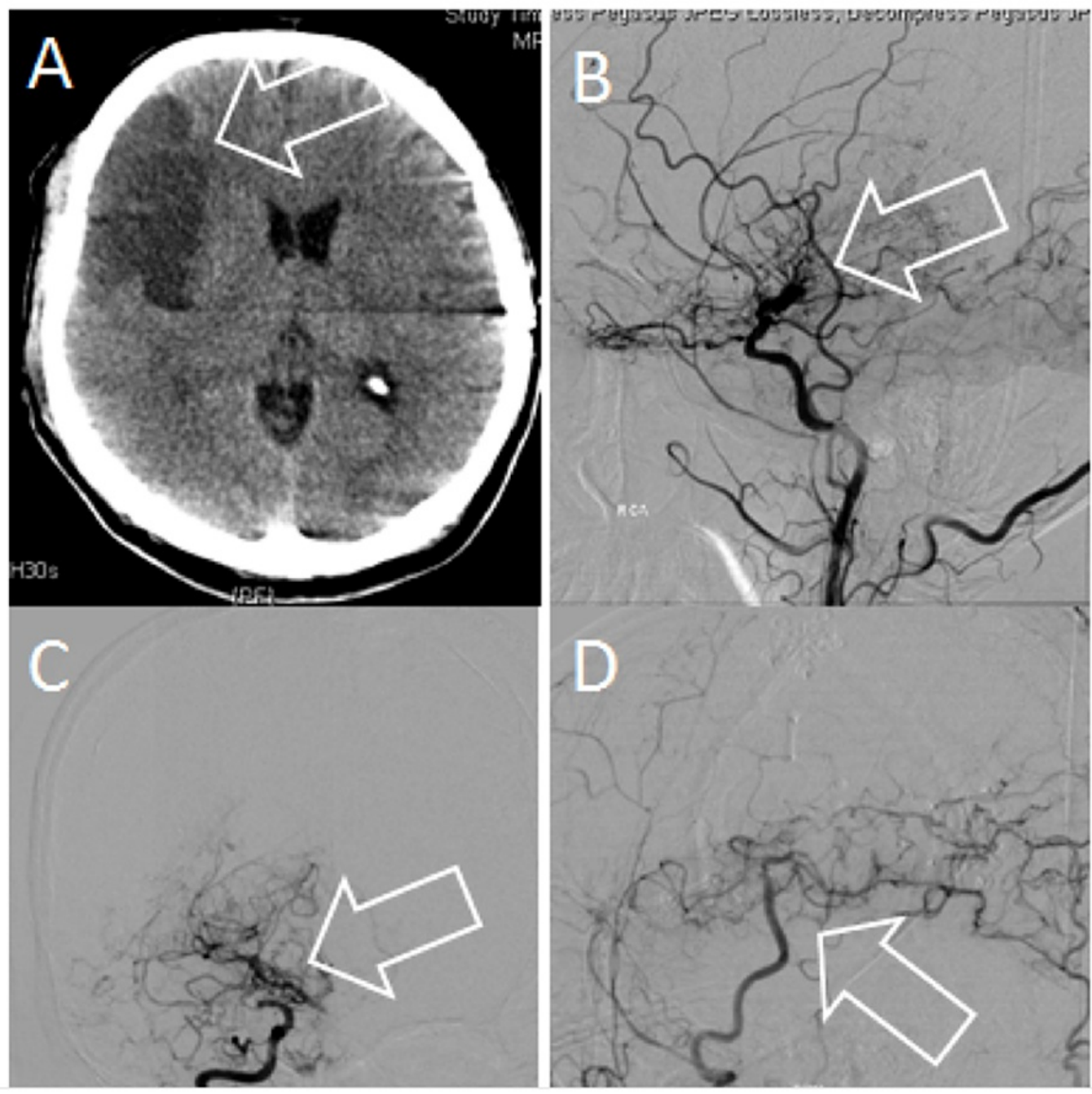

FIGURE 3: This figure illustrates an acute infarction (arrow) on CT

This figure illustrates an acute infarction (arrow) on CT (A) caused by ischemic moyamoya in an adult. Cerebral angiography showed bilateral internal carotid occlusions and extensive fine basal collateral (moyamoya) vessels (B and $C)$. Direct superficial temporal to middle cerebral artery bypass (arrow) with filling of the temporal cortical vessels (D). 


\section{Cureus}

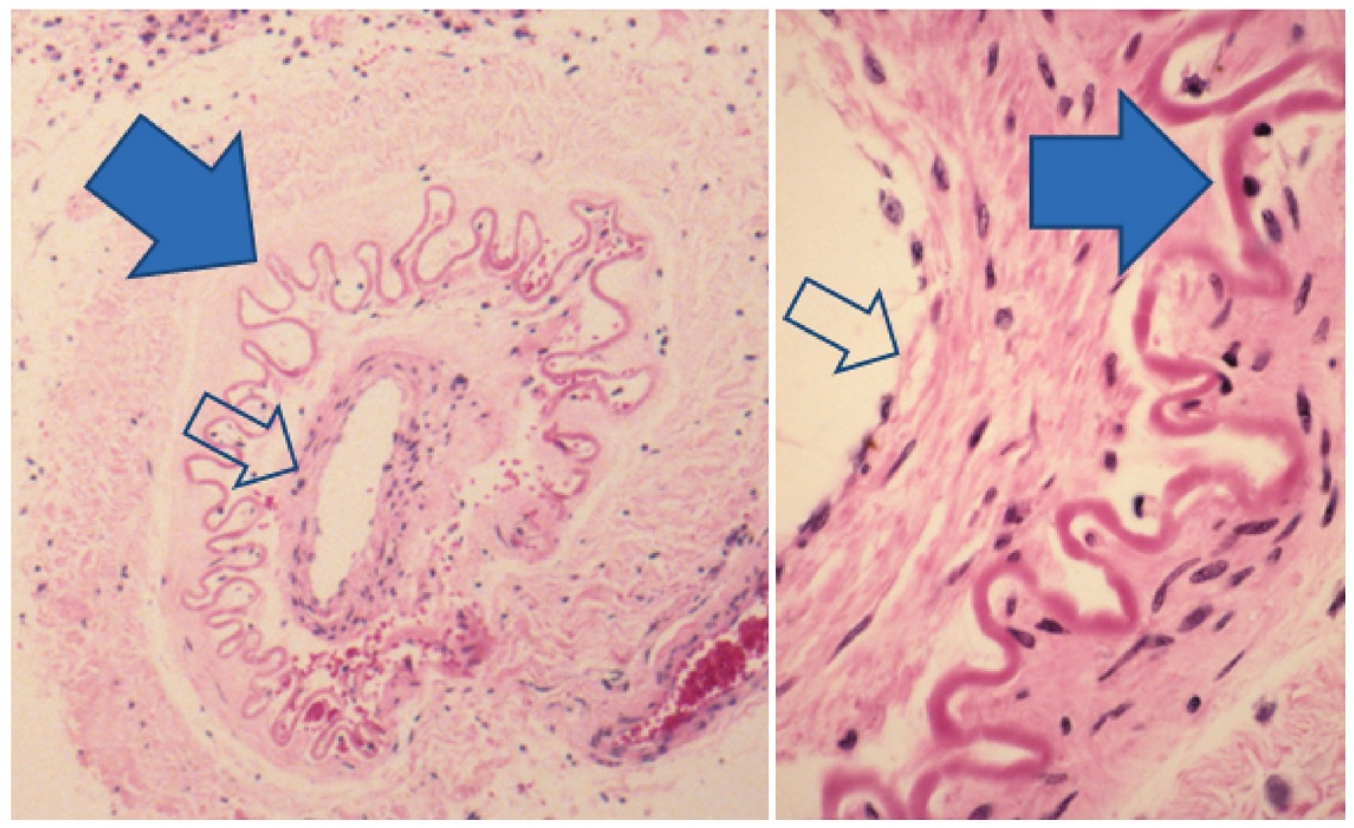

\section{FIGURE 4: Histological findings on hematoxylin and eosin staining of cross section of a large basal artery in a patient with moyamoya}

Histological findings on hematoxylin and eosin staining of cross section of a large basal artery in a patient with moyamoya. Note the fibrosis and thickening of the intima and proliferation of smooth muscle cells (open arrows) and in-folding and chronic contraction of the internal elastic lamina (closed arrows), as well as notable absence of inflammatory or atherosclerotic changes. (Courtesy of Dr Seven Rostad, Swedish Neuroscience Institute, Seattle, Washington.)

The exact cause of the progressive fibrotic changes in the arterial walls of the basal cerebral arteries has been the topic of investigation, and no significant inflammatory or infectious pathogens have been found. Findings from autopsy cases of moyamoya disease have included to a variety of findings [22]. Many of the patients who came to autopsy have done so as a result of intracranial hemorrhage. Hemorrhage occurs usually in the basal ganglia and often can be accompanied by a rupture into the intraventricular space. Subarachnoid hemorrhage can also occur in the basal cisterns and also along the cerebral convexities [24].

The microscopic appearance of the small perforating arteries at the base of the brain and in the parenchyma has also been characterized by abnormal histological changes [23]. Some of these changes indicate flow-related changes in pre-existing but dilated perforating arteries.

Abnormal histological findings have included new vessel formation in response to increased flow demands, including thin walled vessels and microaneurysm formation. These changes occur at the basal vessels and also additional changes occur at the leptomeningeal collateral sites, including findings indicating enhanced capacity for compensatory flow between the external and internal carotid system [46]. These changes include enlargement of the meningeal vessels in the dura with transdural collateral networks as well as transcalvareal collaterals from the scalp vessels which establish enlarged connections to the surface vessels on the brain (Figure 5). A number of investigators have utilized immunohistochemistry of the cerebral vessels and biochemical analysis of CSF to characterize abnormal expression of a number of mediators, including basic fibroblast growth factor (bFGF), transforming growth factor b 1 (TGFb), vascular endothelial growth factor (VEGF), and hypoxia inducible factor (HIF-1) [32, 47- 


\section{Cureus}

51]. It has been recently observed that immunohistochemistry of surgical specimens showed significant increase in serum matrix metalloproteinase-9 expression within the arachnoid membrane of moyamoya [52].
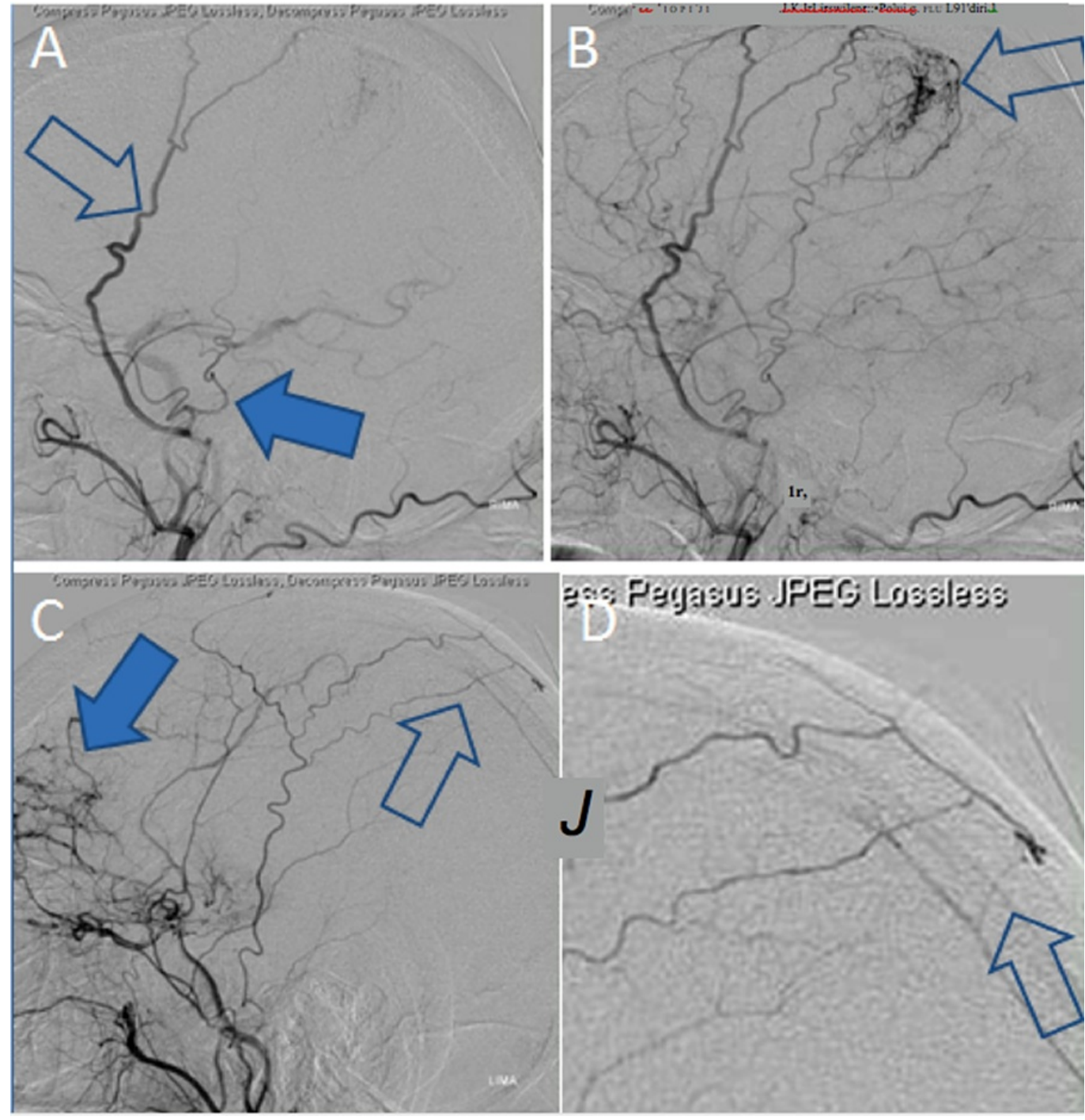

\section{FIGURE 5: Illustration of angiographic findings in late stage ("vault") moyamoya}

Illustration of angiographic findings in late stage ("vault") moyamoya with increase in extracranial carotid collateral vessels supplying blood flow to the cerebral hemispheres through transdural and transcranial collateral vessels. (A) Enlargement of the middle meningeal vessel (open arrow) with (B) transdural collateral vessels, note small superficial temporal artery (A, solid arrow). Contralateral side showing enlarged superficial temporal artery (C, D ,open arrow) supplying transcalvarial branch (through the skull) to supply the cortex, and ethmoidal collaterals (C, solid arrow)

Analysis of cerebrospinal fluid has also shown that a number of mediators involved in the induction of new vessels, including bFGF and VEGF, are increased compared to control patients [53-54]. It has been unclear which vascular growth factors and other cellular mediators expressed in the vessels and CSF of moyamoya patients are related to the primary pathogenic factors causing the basal conducting vessel narrowing, and which are related to secondary 
compensatory changes within the vasculature as a result of ischemia and subsequent collateral network formation [26].

It has been reported that cerebral aneurysms are also associated with moyamoya in some cases [55]. Two types of aneurysms have been described. The first type consists of intracranial aneurysms which form at major arterial bifurcation sites at the circle of Willis, and a second type consists of smaller aneurysms located on moyamoya vessels or enlarged perforating vessels, including choroidal arteries or other enlarged distal cerebral arteries that are serving as collateral vessels. These aneurysms can rupture and cause subarachnoid, intraventricular, or intraparenchymal hemorrhage in moyamoya patients.

The preponderance of evidence indicates that moyamoya is initiated by a genetically and or environmentally-induced idiopathic basal cerebral vessel narrowing and a response of the remaining vasculature to form a collateral network in response to the ischemia and accompanying decrease in cerebral blood flow. Many of the vascular collateral networks seen in moyamoya are known also to occur with basal vessel narrowing caused by other diseases, including intracranial atherosclerosis, radiation induced arteritis, sickle cell anemia [56], and other similar conditions which can produce slow progressive obstructions of the basal intracranial vessels. If the compensatory mechanisms are effective in maintaining blood flow during the progressive basal vessel narrowing and occlusion, then patients may experience either no symptoms or mild or transient ischemic events. If the compensatory mechanisms are insufficient to compensate, then resulting repeated transient or permanent ischemic deficits can take place, with hemodynamic and thrombotic mechanisms both playing a role. The vascular changes which result from the compensatory mechanisms form a collateral vasculature which is more fragile than normal and may predispose to intracranial hemorrhage into the ventricles, parenchyma or subarachnoid space.

\section{Diagnostic imaging studies}

There are a number of diagnostic imaging studies which are used in the diagnosis and management of patients with moyamoya disease. Patients can present with a variety of clinical signs and symptoms, and a number of causes of symptoms; thus, brain imaging can either show ischemic changes or hemorrhage, as well as characteristic vascular changes, which may lead to the suspected diagnosis of moyamoya disease. The confirmation of the diagnosis is made using cerebral angiography which displays the constellation of findings which are required. Diagnostic imaging studies in addition to cerebral angiography which are used commonly in moyamoya include computed tomography (CT), CTA, MR, MRA, Doppler ultrasound, including transcranial Doppler (TCD), as well as extracranial duplex scanning, cerebral blood flow studies including single photon emission computerized tomography (SPECT), CT, and MR perfusion and xenon CT.

\section{Cerebral angiography}

Catheter cerebral angiography remains the gold standard to make the diagnosis of moyamoya. Six vessel cerebral angiography with injection of both carotid arteries, both vertebral arteries as well as selective external carotid injections should be performed in patients with suspected moyamoya. The findings vary depending on the stage of the disease and the extent of the abnormal findings at the time of diagnosis. The classic and essential findings include bilateral narrowing or occlusion of the carotid terminations or "forks" intracranially as well as a blush or puff of smoke in the supraclinoid region indicating hypertrophy and increased number of fine perforating arteries emanating from the basal intracranial vessels bilaterally. Suzuki and Takaku defined six distinct stages of the progression of angiographic findings depending on the degree of advancement of the disease [1]. These stages include narrowing of the carotid fork, initiation of the moyamoya, intensification of the moyamoya, minimization of the moyamoya, 


\section{Cureus}

reduction of the moyamoya, and disappearance of the moyamoya (see Table 2). These stages represent initial narrowing of the basal intracranial arteries, with a reactive increase in perforating artery and posterior circulation collateralization followed by progressive shift of the brain blood supply from the internal carotid to external carotid artery supply, including the middle meningeal and other dural arteries as well as the superficial temporal and other extracranial arteries. The name given to the sum of angiographic changes seen during the late stages of the disease when the blood supply has shifted to the extracranial vessels is called "vault moyamoya." A number of transcalvarial and trans-pial collateral networks develop from the extracranial vessels during this stage of the disease (Figure 5).

\section{Stage Findings}

$1 \quad$ Narrowing of the carotid fork (supraclinoid carotid and proximal middle cerebral and anterior cerebral artery)

2 Initiation of the moyamoya (development of the fine collateral vasculature at the base of the brain) Intensification of the moyamoya (progressive basal vessel narrowing or occlusion with increase in fine collateral vessel formation)

4 Minimization of the moyamoya (basal vessels occluded and decrease in the fine vessel network)

Reduction of the moyamoya (further reduction in basal vessel filling with shift toward extracranial vessel supply)

Disappearance of the moyamoya (occlusion and disappearance of the internal carotid filling of basal and fine collateral vessels bilaterally, with shift to the majority of the blood supply coming from the extracranial vasculature)

\section{TABLE 2: Angiographic stages of moyamoya disease indicating the slow progression over years of the angiographic findings described by Suzuki*}

*Adapted from Suzuki et al 1

Cerebral angiography is important when evaluating patients for potential revascularization therapy. It is important to evaluate the extracranial external carotid artery branches for the suitability for use as donor vessels for extracranial to intracranial bypass procedures. Common findings include enlargement of the middle meningeal branches to form collateral vessels. These vessels sometimes can be confused with the superficial temporal branches (Figure 5).

\section{Magnetic resonance imaging}

An MRI with an MRA scan can often be diagnostic. The combination of these two studies can show acute and chronic parenchymal ischemic changes and acute and chronic posthemorrhagic findings, as well as characteristic changes in the vasculature, including large basal vessel narrowing and dilated moyamoya vessels in the basal ganglia. The moyamoya vessels can sometimes be appreciated as flow voids seen on the T1 axial images (Figures 3, 6). Some patients with poorly characterized past clinical events are found to have moyamoya disease where a number of a small infarctions have already occurred and are usually better seen on $\mathrm{T} 2$ weighted images. The subtle changes on MRI seen in moyamoya disease have been mistaken for multiple sclerosis, other demyelinating or infectious conditions, or hypertensive changes. The initial diagnostic criterion established for moyamoya, required that cerebral angiography be done to establish the diagnosis. Subsequent to the initial criterion, it has been decided that 


\section{Cureus}

MRI can substitute for angiography if the same vascular findings could be discerned with certainty on high quality MRI which meet certain criterion. The use of MRI may spare children and other high risk patients from angiography if the diagnosis is clear on high quality MRI and MRA [24]. These criterions are stated below and include:

The diagnosis of moyamoya disease can be made without conventional cerebral angiography if the MRI and MRA clearly demonstrate all the findings described below that correspond to the diagnostic criteria on conventional cerebral angiography.

1. Stenosis or occlusion is observed at the terminal portions of the intracranial internal carotid arteries and at the proximal portions of the anterior communicating arteries and the middle cerebral arteries on MRA.

2. Visualization of abnormal vascular networks in the basal ganglia on MRA, or demonstration of moyamoya vessels as at least two apparent signal voids in the ipsilateral side of the basal ganglia on MRI.

\section{These two findings are found bilaterally.}

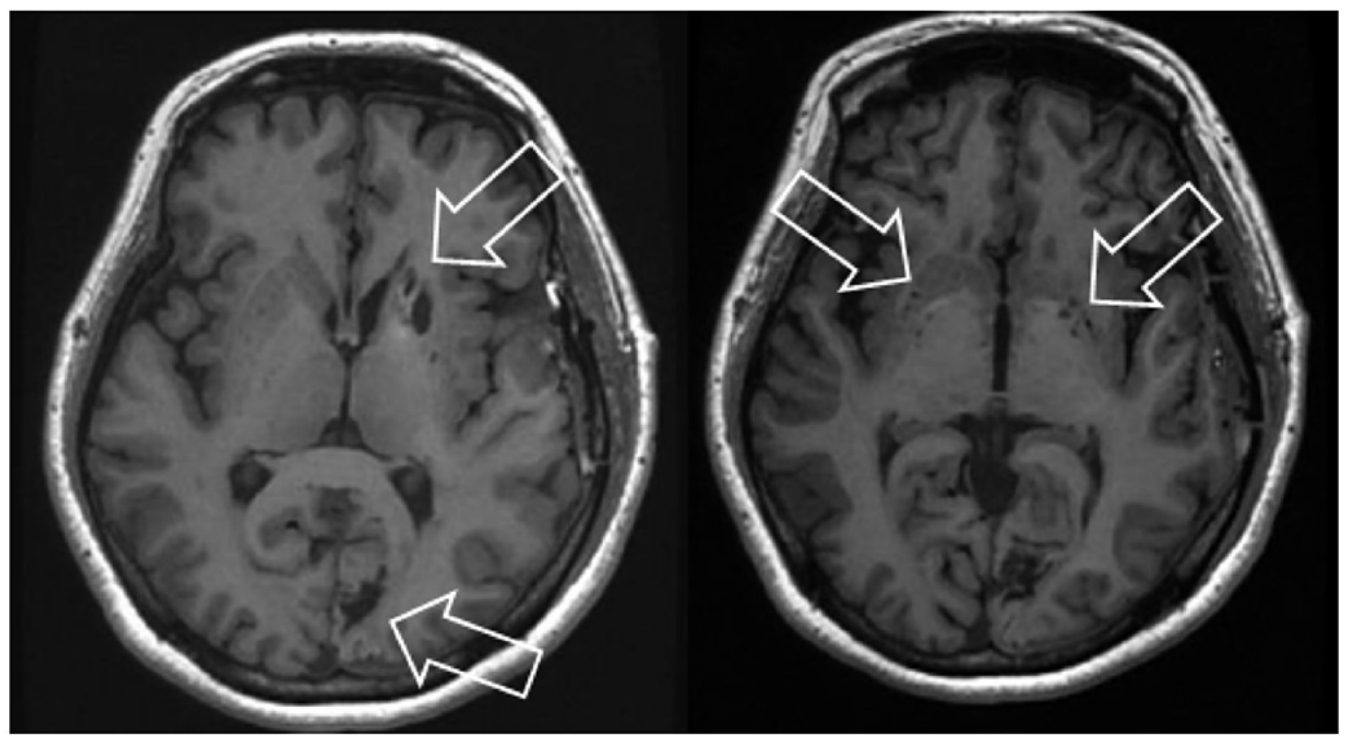

FIGURE 6: Illustration of findings consistent with moyamoya on T1 weighted MRI scan

Illustration of findings consistent with moyamoya on T1 weighted MRI scan showing evidence of old infarctions (arrows) as a result on ischemic moyamoya in an adult (left) multiple flow voids (right) indicating moyamoya collateral vessels in the basal ganglia.

\section{Imaging methods and limitations include:}

1. The use of magnetic resonance machine with a static magnetic field strength of 1.0 tesla or stronger is recommended.

2. Any method for obtaining the images of MRA can be used.

3. A static magnetic field strength, the imaging method, and the use of contrast medium should 
be used.

\section{Computerized tomography}

Plain CT scan can often be useful as a first diagnostic test if patients present to medical attention with new deficits, and can reveal subarachnoid, intraventricular, or intraparenchymal hemorrhage, or infarction in patients with moyamoya. It can also be useful in determining whether calcium deposits are present in the vessels in cases where atherosclerosis is in the differential diagnosis as a cause of vessel narrowing. In some cases, old areas of infarction or hemorrhage may also be revealed. During clinical presentation of the hemorrhagic form, CTA may be combined with CT to rule out a vascular lesion as a cause of the intracranial hemorrhage, and can sometimes reveal the characteristic changes of moyamoya in the vasculature (see Figure 2). CT perfusion and xenon CT may also be used to determine blood flow changes in moyamoya and be used as a guide for therapy. Another use of CTA is to help determine graft patency on follow-up of patients who have had revascularization procedures, and to determine if there has been any progression of disease in patients who presented with unilateral disease and are at risk for progression [57].

\section{Doppler ultrasound}

Cerebrovascular ultrasound studies, including carotid ultrasound and transcranial Doppler ultrasound, may be useful in the diagnosis and management of patients with moyamoya [31, 58-60]. If patients are initially screened for central nervous system symptoms, including transient ischemic events, findings on Doppler ultrasonography may strongly suggest the diagnosis of moyamoya. Characteristic carotid duplex scan findings include the decreased flow velocity with high resistance in the internal carotid arteries in the cervical region as well as the higher velocity with the lower resistance findings in the external carotid artery system. Transcranial Doppler findings often include diffuse bilateral high velocity signals in the basal intracranial arteries resulting from a stenosis or occlusion of the basal cerebral arteries and high velocity in collateral vessels, in cases with more advanced progression. Reduced mean velocity and reduced vascular reactivity in the more distal portions of the middle cerebral artery distal branches has also been noted. TCD has also been useful to demonstrate graft patency in the follow up of patients with moyamoya [61]. One other application of TCD is to follow the progression of intracranial basal artery velocity in patients who have findings of moyamoya on one side and are at risk for progression of the asymptomatic side.

\section{Cerebral blood flow studies}

Cerebral blood flow (CBF) studies have been useful in documenting the final effect of the collateral networks in moyamoya to support cerebral blood flow in patients with ischemic symptoms. Resting CBF measurements and $\mathrm{CBF}$ changes in response to acetazolamide or $\mathrm{CO}_{2}$ can be used to evaluate cerebrovasular reserve. There are a number of CBF studies which have become more widely available for clinical testing in moyamoya patients, and can be used for regional CBF comparisons as well as vascular reactivity studies [62-68]. These include single photon emission computerized tomography (SPECT), CT perfusion, and MR perfusion. Quantitative methods which are available mainly on a research basis include quantitative xenon CT and positron emission tomography (PET). These studies the may be helpful in defining which hemispheres are more at risk for immediate ischemic changes in patients with bilateral vascular changes in moyamoya disease. Often, the degree of narrowing of the vessels at the base of the brain is not necessarily indicative of the degree of ischemia which occurs in the cerebral hemispheres. Some of the variations of the effectiveness of the collateral networks can often account for quite different CBF findings in individual patients with moyamoya.

The determination of a regional CBF changes may be helpful in directing the sequence of 
therapy which is chosen and also to measure the effectiveness of therapies on correcting blood flow deficits $[9,69]$.

\section{Elecroencephalography}

Patients with moyamoya can have overt seizures as a presentation and display continued symptomatology, although seizures as a presentation are less common than ischemic and hemorrhagic symptoms. Abnormal electroencephalograms (EEG) are more commonly found in children with moyamoya than in adults. Some of the findings which have been described include bilateral low voltage changes and slow waves, and delta waves during hyperventilation [70].

\section{Natural history of moyamoya disease}

Most natural history studies of moyamoya disease have been retrospective in nature and limited to small groups of patients. Many of the studies published have separated pediatric and adult patients. Several studies were performed on pediatric patients who were treated conservatively and have recorded the development of subsequent clinical events [71-72]. Kurokawa, et al. reported that about $80 \%$ of pediatric patients followed out for five years without surgical treatment developed subsequent ischemic or hemorrhagic symptoms. Ezura, et al. followed patients who were diagnosed as pediatric moyamoya into adulthood [73]. These investigators have indicated that neurologic events occurred in 11/23 and mental disorders occurred in 9/21 patients who were followed into adolescence and adulthood.

In adults, the natural history studies to date have indicated that the prognosis is poor for those symptomatic adults treated conservatively without surgery. There are few data reported in cohorts of patients who have had no surgical treatment at all and have been followed. Most cohorts have some patients where one hemisphere is treated surgically and the other was followed, or mixed cohorts where some patients were treated with surgery and others were not. Kuroda, et al. have reported a series of Japanese patients followed over a long period of time where some patients were not treated with surgery [74]. In this cohort study of 120 adult Japanese patients diagnosed with moyamoya disease, 63 patients were enrolled in this historical prospective cohort study which included a total of 86 non-operated hemispheres. All patients were followed up with a mean period of 73.6 months. Patients were evaluated by MRI and MRA imaging which was repeated every six to 12 months. Cerebral angiography was performed when disease progression was suspected on MRI and MRA. Disease progression occurred in 15 of the 86 non-operated hemispheres (17.4\% per hemisphere) or in 15 of 63 patients (23.8\% per patient) during the mean six year follow-up period. Occlusive arterial lesions progressed in both anterior and posterior circulations, in both symptomatic and asymptomatic patients, and in both bilateral and unilateral types. Eight of 15 patients developed ischemic or hemorrhagic events related to disease progression.

Hallemeier, et al. reported a high recurrent stroke risk in non-operated hemispheres in a group of North American patients [28]. Thirty-four adults (median age 42) with moyamoya treated at a single North American institution were followed prospectively. Twenty-two had bilateral involvement and 12 had unilateral moyamoya vessels. The initial symptom was ischemia, hemorrhage, or asymptomatic in 24 , seven, and three patients, respectively. The median follow-up in 31 living patients was 5.1 years. Fourteen patients were treated with surgical revascularization (20 total hemispheres). In medically treated symptomatic hemispheres, the five-year risk of recurrent ipsilateral stroke was $65 \%$ after the initial symptom and $27 \%$ after angiographic diagnosis. Patients with bilateral involvement presenting with ischemic symptoms $(n=17)$ were at the highest risk of subsequent stroke, with a five-year risk of stroke with medical treatment after first symptom of $82 \%$. In surgically treated hemispheres, the fiveyear risk of perioperative or subsequent ipsilateral stroke or death was $17 \%$. This stroke and 
death rate was significantly lower than the patients who received medical treatment after the first symptom $(\mathrm{P}=0.02)$ but not lower than the group of patients who presented after angiographic diagnosis without symptoms.

In a series from Japan of patients presenting with hemorrhagic moyamoya, a retrospective review was conducted among 42 patients treated conservatively without bypass surgery and followed for an average period of 80.6 months [75]. The group included four patients who had undergone indirect bypass surgery after an episode of rebleeding. Initial presentation was intraventricular hemorrhage with or without intracerebral hemorrhage during the first bleeding episode in 29 cases (69\%). During the follow-up period, 14 patients experienced a second episode of bleeding. The annual re-bleeding rate was 7.09\%/person/year. Recurrent hemorrhages occurred in the original site in some patients and at new sites in others. Another series was reported by Yoshida, et al., which suggested that revascularization may reduce rebleeding episodes [76]. In patients who presented with bleeding episodes, five of the 28 patients (17.9\%) died of the initial intracranial hemorrhage, and two patients died of other causes. Rebleeding occurred in six of the remaining 21 patients (28. 6\%). Of these six patients, four died of rebleeding. Rebleeding occurred in one of eight patients who underwent bypass surgery and in five of 13 patients who did not, which suggested that rebleeding was less likely to occur in patients who had undergone bypass surgery. However, the results did not reach statistical significance $(\mathrm{P}>0.05)$. Kawaguchi, et al. reported another cohort study which compared the effect of direct bypass with indirect bypass or conservative treatment to prevent further ischemic or hemorrhagic stroke in patients initially presenting with hemorrhage [77]. From a cohort of twenty-two patients who had hemorrhagic moyamoya disease, eleven patients (50\%) were conservatively treated. Among the 11 patients who were surgically treated, STAMCA bypass was performed in six patients (27\%) and encephaloduroarteriosynangiosis (EDAS) in the other five patients (23\%). Nine patients (41\%) presented with an ischemic or re-bleeding event during the follow-up period. The incidence of future stroke events in patients who had undergone an STA-MCA bypass was significantly lower $(\mathrm{p}<0.05)$ than that in patients who had been treated conservatively or with EDAS. Stroke-free times in patients treated with direct bypass and those in patients who conservatively or with indirect bypass showed a significant difference Kaplan-Meier plots $(\mathrm{p}<0.05)$ in favor of direct bypass.

One of the current controversies in managing moyamoya patients is regarding the degree of intervention which is appropriate when patients have presented with vascular changes which are limited to one cerebral hemisphere. According to established criterion, these patients would be considered as having a diagnosis of probable moyamoya disease and are sometimes referred to as having moyamoya syndrome. Smith, et al. reviewed the clinical and imaging records of a series of primarily pediatric patients with moyamoya syndrome who presented with unilateral disease and underwent cerebral revascularization surgery using pial synangiosis, or EDAS, on the affected side [57]. In a group of 235 surgically treated patients with moyamoya, 33 (14\%) presented with unilateral disease (four adults and 29 children). There were 16 female and 17 male patients, with an average age of 10.4 years ( 26.8 years for adults and 8.1 years for children; range 1.5-39 years). The average follow-up after surgery was 5.3 years (3.1 years for adults and 5.6 years for children; range 1-16 years). During the follow-up period, $10(30 \%)$ of 33 patients with unilateral findings, progressed to bilateral disease as evidenced by appearance of moyamoya changes in the previously unaffected hemisphere. The mean time until disease progression was 2.2 years (range $0.5-8.5$ years). Certain factors were associated with progression including contralateral abnormalities on initial angiography, previous history of congenital cardiac anomaly, cranial irradiation, Asian ancestry, and familial moyamoya syndrome. It was therefore recommended that pediatric patients with known unilateral disease should undergo continued monitoring by diagnostic testing at regular intervals.

\section{Medical treatment}


A number of medical treatments have been tried for patients with moyamoya. Antiplatelet agents, most commonly acetylsalicylic acid, are routinely used for patients presenting with ischemic symptoms with the rationale that vascular thrombosis in the cerebral arteries is commonly observed. Anticoagulation using warfarin is not commonly used and may be contraindicated due to the propensity for intracranial hemorrhage in moyamoya. Other medications, including vasodilators, anticonvulsants, and fibrinolytics, have also been used but no controlled trials and no good cohort studies have provided strong support for clinical effectiveness of any medications.

\section{Surgical treatment}

Surgical revascularization as a treatment for moyamoya disease was first proposed described by Yassargil and colleagues [78-79] and subsequent adapted to moyamoya by Karasawa and Kikuchi [80]. There are two types of revascularization surgery that are now practiced for the treatment of a moyamoya disease and are termed indirect and direct revascularization.

\section{Indirect revascularization}

Indirect revascularization involves placing vascularized tissue fed by the external carotid system on to the surface of the brain to allow collateral networks to form and reroute blood flow around the obstructed vessels at the base of the brain. A number of different tissues have been proposed and tried, including muscle, omentem, galea, and dura. The most commonly practiced technique currently is termed encephaloduroarteriosynangiosis where the superficial temporal artery is kept flowing in-situ, and dissected along with vascularized galea which is then sewn into an opening in the dura and is placed in contact with the cerebral cortex as described by Matsushima et al. [81-82] (see Figure 7). This procedure is favored in most centers for pediatric patients due to the small size of the vessels which make direct anastomosis difficult. Indirect techniques are also utilized in adults and can be useful when the donor or recipient vessels are of insufficient size to allow direct anastomosis [6, 13-14, 83-89]. 


\section{Cureus}
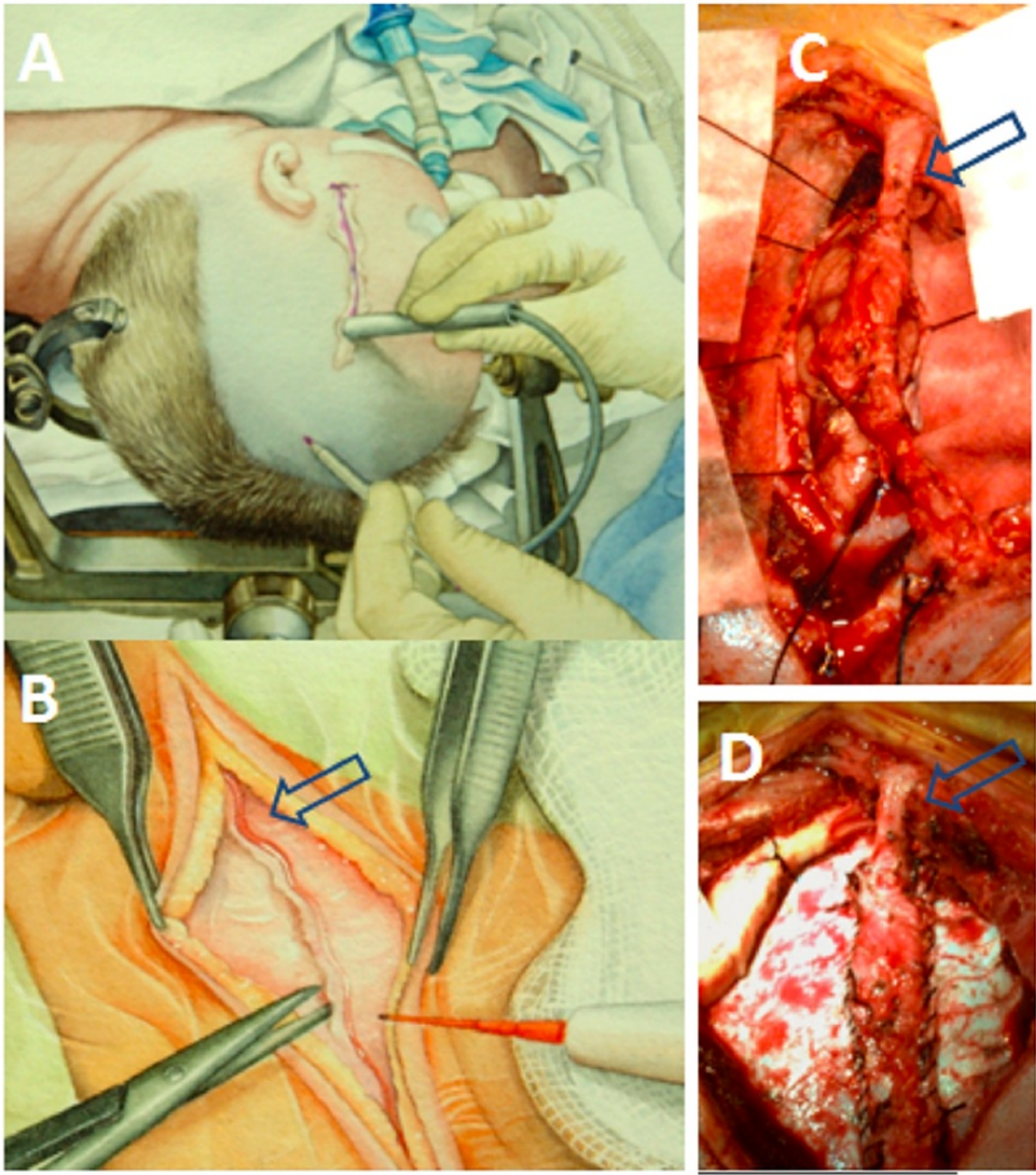

FIGURE 7: Drawing and intra-operative photograph of

\section{dissection and preparation of the superficial temporal artery with surrounding galeal pedicle $(A, B)$}

Drawing and intra-operative photograph of dissection and preparation of the superficial temporal artery with surrounding galeal pedicle $(A, B)$. Dural opening and apposition of vascularized pedicle onto the cerebral cortical surface for encephaloduroarteriosynangiosis procedure (C). The superficial temporal artery (arrows) continues to flow, and the artery and galeal pedicle are sutured into the dural opening (D) and provides a source of in-growth of collateral vessels to the leptomeninges and supplies additional blood flow to the middle cerebral artery territory after a maturation period.

\section{Direct revascularization}

The second treatment is termed direct revascularization, where a direct microvasular anastomosis between the extracranial and intracranial vessels is performed. The most common procedure performed is a direct superficial temporal to middle cerebral artery bypass (see Figure 8) using a microvasular suture technique [90]. A microvasular anastomosis technique has 


\section{Cureus}

also been described for direct anatomosis of the superficial temporal to middle cerebral artery in moyamoya patients [91]. In cases where these arteries are not suitable, other direct vascular anastomosis techniques have been employed using other arteries or vein grafts [91-92]. The rationale for this treatment is to allow the more rapid development of collateral vessels to ischemic portions of the brain to prevent recurrent TIAs and/or stroke. Several comparison studies between the direct and the indirect techniques have been performed. In patients with reduced $\mathrm{CBF}$, the direct procedure is often more effective for a more rapid increase in blood flow to the affected brain region [91, 93-98].
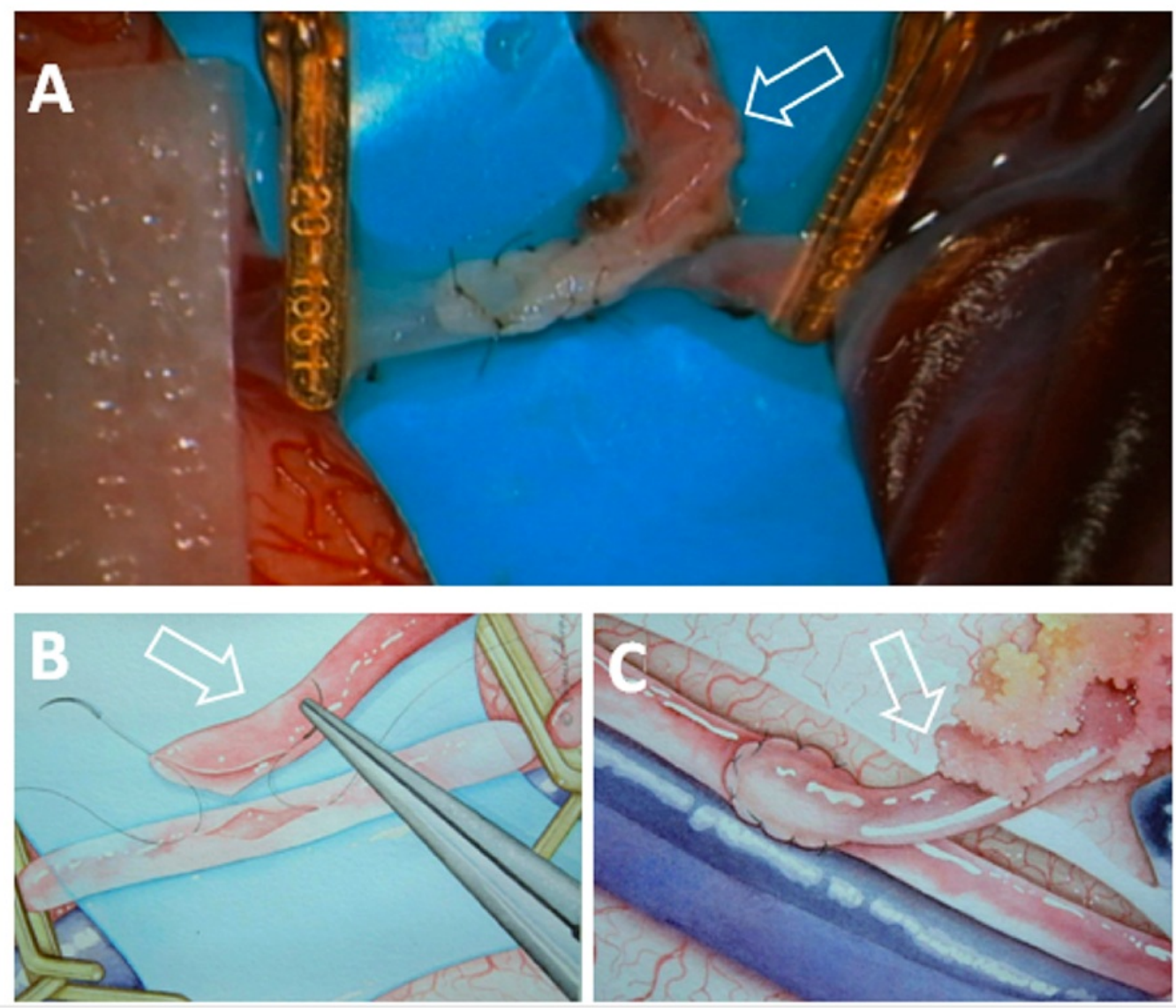

FIGURE 8: Drawings (A, B) and intra-operative photograph (C) of direct superficial temporal (arrows) to middle cerebral artery bypass

Drawings (A, B) and intra-operative photograph (C) of direct superficial temporal (arrows) to middle cerebral artery bypass using a microsuture technique to accomplish an end to side microvascular anastomosis.

\section{Long-term effect of revascularization for moyamoya disease}

Despite the clinical differences between US, European and Asian moyamoya populations, it appears that the benefits of revascularization procedures are similar. The progressive obliteration of the intracranial arterial supply in moyamoya disease decreases cerebral perfusion, producing ischemic episodes and exhausting vasomotor reserve. As abnormal collaterals form to provide additional blood supply to the brain, cerebral autoregulation and perfusion dynamics are further altered. Analysis of CBF and hemodynamics in these patients 
following revascularization operations have demonstrated marked improvements in these parameters. Following direct STA-MCA bypass in six children, Yonekawa, et al. [19] noted that rCBF improved in both the operated and contralateral hemispheres. Okada, et al. [99] demonstrated similar findings for $\mathrm{rCBF}$ and normalization of cortical perfusion pressure in adult patients following direct STA-MCA bypasses. Our results, while in a population with different demographics, echo these earlier findings in general [9]. In our cohort, cerebral perfusion and vasomotor reactivity improved in all patients who underwent postoperative blood flow and TCD studies. Of particular interest, five patients who had suffered only TIAs experienced a complete normalization of cerebral perfusion and vasomotor reactivity following their bypass procedures. This effect appeared durable in the long-term, as they had been asymptomatic throughout the follow-up period (average 43 months).

In contrast to Asian series that demonstrate a complete resolution of ischemic symptoms following bypass operations, infrequent ischemic episodes occurred in our patients. These events occurred in six patients who suffered TIAs on a weekly basis prior to their bypass. One patient suffered a TIA on the first postoperative day and then was event-free for the following 48 months. Another patient experienced a TIA one week postoperatively and was event free for the following seven years. The remaining four patients suffered TIAs 12- 48 months postoperatively.

Other published series of revascularization procedures have not demonstrated a significant correlation between reduction in moyamoya vessels and prevention of hemorrhagic events. In fact, reduction in moyamoya vessels is observed in only $25-65 \%$ of patients. Moreover, the ability of surgery to prevent or reduce the frequency of hemorrhagic events has been disappointing. Okada, et al. [99] reported that $20 \%$ of patients who presented with hemorrhagic events suffered additional hemorrhagic events following direct STA-MCA bypass. Yonekawa, et al.[19] reported that the rate of rebleeding following surgery was no different than the natural history of the disease, although other reports indicate that there may be a protective effect of revascularization [77].

Surgical revascularization for the treatment of moyamoya disease may provide long-term reduction in ischemic events compared to the natural history, although most current data is based on observational studies [25-26]. Regardless of the differences that exist between the demographic characteristics of moyamoya patients in the Western and Eastern hemispheres, improvement in cerebral perfusion and vasomotor reactivity can be expected, correlating to a reduction in ischemic events with revasularization treatment. Further investigations are required to determine the etiology of hemorrhagic events in moyamoya disease, and a larger population with the hemorrhagic variety of moyamoya disease must be evaluated to clarify whether surgical revascularization alters the frequency of these events.

\section{Conclusions}

Moyamoya disease has become increasingly recognized as a cause of hemorrhagic and ischemic stroke which can affect children and adults in Western populations as well as in Asia. Advances in neuroimaging have led to an increased recognition of the disease worldwide. As more information is gathered, the details of the natural history as well as the clinical and physiologic effects of treatments are becoming more clearly understood. Surgical revascularization using either the indirect or direct method is widely used for the treatment of moyamoya. The use of revascularization for the treatment of pediatric and adult patients presenting with ischemic symptoms is supported by multiple cohort studies, but its efficacy has not been established by randomized controlled trials. Although the cause of the disease is not understood, the available information indicates that both genetic and environmental factors play a significant role. It is expected that with advances in molecular biology and genetics, the molecular mechanisms underlying the disease and its progression will be better understood and eventually lead to 
further advances in treatments.

\section{Additional Information}

\section{Disclosures}

Conflicts of interest: In compliance with the ICMJE uniform disclosure form, all authors declare the following: Payment/services info: All authors have declared that no financial support was received from any organization for the submitted work. Financial relationships: All authors have declared that they have no financial relationships at present or within the previous three years with any organizations that might have an interest in the submitted work. Other relationships: All authors have declared that there are no other relationships or activities that could appear to have influenced the submitted work.

\section{References}

1. Suzuki J, Takaku A: Cerebrovascular "moyamoya" disease. Disease showing abnormal net-like vessels in base of brain. Arch Neurol. 1969, 20:288-299.

2. Kikuta K, Takagi Y, Arakawa Y, et al.: Absence epilepsy associated with moyamoya disease. A case report.. J Neurosurg. 2006, 104:265-268.

3. Ise $\mathrm{H}$, Tokunaga $\mathrm{H}$, Aizawa M, Sawaura $\mathrm{H}$ : Cerebellar hemorrhage associated with moyamoya disease. Neurol Med Chir (Tokyo). 1998, 38:225-228.

4. Andreone V, Ciarmiello A, Fusco C, Ambrosanio G, Florio C, Linfante I: Moyamoya disease in Italian monozygotic twins. Neurology. 1999, 53:1332-1335.

5. Edwards-Brown MK, Quets JP: Midwest experience with moyamoya disease. Clin Neurol Neurosurg. 1997, 99:36-38.

6. Golby AJ, Marks MP, Thompson RC, Steinberg GK: Direct and combined revascularization in pediatric moyamoya disease. Neurosurgery. 1999, 45:50-58.

7. Goto Y, Yonekawa Y: Worldwide distribution of moyamoya disease . Neurol Med Chir (Tokyo). 1992, 32:883-886.

8. Kraemer M, Heienbrok W, Berlit P: Moyamoya disease in Europeans . Stroke. 2008, 39:31933200. 10.1161/STROKEAHA.107.513408

9. Mesiwala AH, Sviri G, Fatemi N, Britz GW, Newell DW: Long-term outcome of superficial temporal artery-middle cerebral artery bypass for patients with moyamoya disease in the US. Neurosurg Focus. 2008, 24:15. 10.3171/FOC/2008/24/2/E15

10. Minelli C, Takayanagui OM, dos Santos AC, Fabio SC, Lima JE, Sato T, Colli BO: Moyamoya disease in Brazil. Acta Neurol Scand. 1997, 95:125-128.

11. Olds MV, Griebel RW, Hoffman HJ, Craven M, Chuang S, Schutz H: The surgical treatment of childhood moyamoya disease. J Neurosurg. 1987, 66:675-680.

12. Peerless SJ: Risk factors of moyamoya disease in Canada and the USA. . Clin Neurol Neurosurg. 1997, 99:45-48.

13. Scott RM: Surgical treatment of moyamoya syndrome in children. 1985 . Pediatr Neurosurg. 1995, 22:39-46.

14. Scott RM, Smith JL, Robertson RL, et al: Long-term outcome in children with moyamoya syndrome after cranial revascularization by pial synangiosis.. J Neurosurg. 2004, 100:142-149.

15. Srinivasan J, Britz GW, Newell DW: Cerebral revascularization for moyamoya disease in adults. Neurosurg Clin N Am. 2001, 12:585-594.

16. Vörös E, Kiss M, Hankó J, Nagy E: Moyamoya with arterial anomalies: relevance to pathogenesis. Neuroradiology. 1997, 39:852-856.

17. Wetjen NM, Garell PC, Stence NV, Loftus CM: Moyamoya disease in the midwestern United States. Neurosurg Focus. 1998, 5:

18. Williams DL, Martin IL, Gully RM: Intracerebral hemorrhage and Moyamoya disease in pregnancy. Can J Anaesth. 2000, 47:996-1000.

19. Yonekawa Y, Ogata N, Kaku Y, et al.: Moyamoya disease in Europe, past and present status. . Clin Neurol Neurosurg . 1997, 99:58-60.

20. Zafeiriou DI, Ikeda H, Anastasiou A, et al: Familial moyamoya disease in a Greek family . Brain Dev. 2003, 25:288-290. 
21. Takeuchi K, Shimizu, K: Hypoplasia of the bilateral internal carotid arteries . Brain Nerve. 1957, 9:37-43.

22. Hosoda Y, Ikeda E, Hirose S: Histopathological studies on spontaneous occlusion of the circle of Willis (cerebrovascular moyamoya disease). Clin Neurol Neurosurg. 1997, 99:2003-208.

23. Yamashita M, Oka K, Tanaka K: Histopathology of the brain vascular network in moyamoya disease. Stroke . 1983, 14:50-58.

24. Fukui M: Guidelines for the diagnosis and treatment of spontaneous occlusion of the circle of Willis ('moyamoya' disease). Research Committee on Spontaneous Occlusion of the Circle of Willis (Moyamoya Disease) of the Ministry of Health and Welfare, Japan. Clin Neurol Neurosurg. 1997, 99:238-240.

25. Kuroda S, Houkin K: Moyamoya disease: current concepts and future perspectives. Lancet Neurol. 2008, 7:1056-1066. 10.1016/S1474-4422(08)70240-0

26. Scott RM, Smith ER: Moyamoya disease and moyamoya syndrome. N Engl J Med. 2009, 360:1226-1237. 10.1056/NEJMra0804622

27. Chiu D, Shedden P, Bratina P, et al: Clinical features of moyamoya disease in the United States. Stroke . 1998, 29:1347-1351.

28. Hallemeier CL, Rich KM, Grubb RL Jr., et al: Clinical features and outcome in North American adults with moyamoya phenomenon. Stroke. 2006, 37:1490-1496.

29. Uchino K, Johnston SC, Becker KJ, et al: Moyamoya disease in Washington State and California. Neurology. 2005, 65:956-958.

30. Khan N, Yonekawa Y: Moyamoya angiopathy in Europe. Acta Neurochir Suppl. 2005, 94:149152.

31. Laborde G, Harders A, Klimek L, et al: Correlation between clinical, angiographic and transcranial Doppler sonographic findings in patients with moyamoya disease. Neurol Res. 1993, 15:87-92.

32. Achrol AS, Guzman R, Lee M, et al: Pathophysiology and genetic factors in moyamoya disease . Neurosurg Focus. 2009, 26:10.3171/2009.1.FOCUS08302

33. Wakai K, Tamakoshi A, Ikezaki K, et al: Epidemiological features of moyamoya disease in Japan: findings from a nationwide survey. Clin Neurol Neurosurg. 1997, 99:1-5.

34. Tanghetti B, Capra R, Giunta F, et al: Moyamoya syndrome in only one of two identical twins. Case report. J Neurosurg. 1983, 59:1092-1094.

35. Mineharu Y, Liu W, Inoue K, et al: Autosomal dominant moyamoya disease maps to chromosome 17q25.3.. Neurology. 2008, 70:2357-2363. 10.1212/01.wnl.0000291012.49986.f9

36. Aoki N: Moyamoya syndrome in twins. J Neurosurg. 1984, 61:617.

37. Duman Y, Dirlik A, Burak Z, et al: Moyamoya disease in twins . Clin Nucl Med. 1995, 20:810812.

38. Ikeda H, Sasaki T, Yoshimoto T, Fukui M, Arinami T: Mapping of a familial moyamoya disease gene to chromosome 3p24.2-p26.. Am J Hum Genet. 1999, 64:533-537.

39. Ikeda H, Yoshimoto T: Specific genetic characteristics in patients with familial moyamoya disease. J Stroke Cerebrovasc Dis. 2005, 14:244-250.

40. Inoue TK, Ikezaki K, Sasazuki T, et al: Linkage analysis of moyamoya disease on chromosome 6. J Child Neurol. 2000, 15:179-182.

41. Koc F, Yerdelen D, Koc Z: Neurofibromatosis type 1 association with moyamoya disease . Int J Neurosci. 2008, 118:1157-1163. 10.1080/00207450801898279

42. Meschia JF, Ross OA: Heterogeneity of Moyamoya disease: after a decade of linkage, is there new hope for a gene?. Neurology. 2008, 70:2353-2354. 10.1212/01.wnl.0000314695.96436.19

43. Mineharu Y, Takenaka K, Yamakawa H, et al: Inheritance pattern of familial moyamoya disease: autosomal dominant mode and genomic imprinting. J Neurol Neurosurg Psychiatry. 2006, 77:1025-1029.

44. Yamauchi T, Tada M, Houkin K, et al: Linkage of familial moyamoya disease (spontaneous occlusion of the circle of Willis) to chromosome 17q25. Stroke. 2000, 31:930-935.

45. Fukui M, Kono S, Sueishi K, Ikezaki K: Moyamoya disease. Neuropathology. 2000, 20:61-64.

46. Kono S, Oka K, Sueishi K: Histopathologic and morphometric studies of leptomeningeal vessels in moyamoya disease. Stroke . 1990, 21:1044-1050.

47. Hojo M, Hoshimaru M, Miyamoto S, et al: Role of transforming growth factorbetal in the pathogenesis of moyamoya disease. J Neurosurg. 1998, 89:623-629.

48. Houkin K, Yoshimoto $\mathrm{T}$, Abe H, et al: Role of basic fibroblast growth factor in the pathogenesis of moyamoya disease. Neurosurg Focus. 1998, 5: 
49. Li B, Wang CC, Zhao ZZ, et al: A histological, ultrastructural and immunohistochemical study of superficial temporal arteries and middle meningeal arteries in moyamoya disease. Acta Pathol Jpn. 1991, 41:521-530.

50. Terai Y, Kamata I, Ohmoto T: Experimental study of the pathogenesis of moyamoya disease: histological changes in the arterial wall caused by immunological reactions in monkeys. Acta Med Okayama. 2003, 57:241-248.

51. Yamamoto M, Aoyagi M, Tajima S, et al: Increase in elastin gene expression and protein synthesis in arterial smooth muscle cells derived from patients with Moyamoya disease. Stroke. 1997, 28:1733-1738.

52. Fujimura M, Watanabe M, Narisawa A, et al: Increased expression of serum matrix metalloproteinase-9 in patients with moyamoya disease. Surg Neurol. 2009, 72:476-480. 10.1016/j.surneu.2008.10.009

53. Burke GM, Burke AM, Sherma AK, et al: Moyamoya disease: a summary. Neurosurg Focus. 2009, 26:10.3171/2009.1.FOCUS08310

54. Yoshimoto T, Houkin K, Takahashi A, et al.: Evaluation of cytokines in cerebrospinal fluid from patients with moyamoya disease. Clin Neurol Neurosurg. 1997, 99:218-220.

55. Konishi Y, Kadowaki C, Hara M, et al: Aneurysms associated with moyamoya disease . Neurosurgery. 1985, 16:484-491.

56. Smith ER, McClain CD, Heeney M, et al: Pial synangiosis in patients with moyamoya syndrome and sickle cell anemia: perioperative management and surgical outcome. Neurosurg Focus. 2009, 26:10.3171/2009.01.FOCUS08307

57. Smith ER, Scott RM: Progression of disease in unilateral moyamoya syndrome . Neurosurg Focus. 2008, 24:10.3171/FOC/2008/24/2/E17

58. Halpern EJ, Nack TL: Prospective diagnosis of moyamoya disease with Doppler ultrasonography. J Ultrasound Med. 1995, 14:157-160.

59. Iguchi Y, Kimura K, Tateishi Y, et al: Microembolic signals are associated with progression of arterial lesion in Moyamoya disease: a case report. J Neurol Sci. 2007, 260:253-255.

60. Ruan LT, Duan YY, Cao TS, et al: Color and power Doppler sonography of extracranial and intracranial arteries in Moyamoya disease. J Clin Ultrasound. 2006, 34:60-69.

61. Perren F, Meairs S, Schmiedek P, et al: Power Doppler evaluation of revascularization in childhood moyamoya. Neurology. 2005, 64:558-560.

62. McAuley DJ, Poskitt K, Steinbok P: Predicting stroke risk in pediatric moyamoya disease with xenon-enhanced computed tomography. Neurosurgery. 2004, 55:327-332.

63. Mountz JM, Foster NL, Ackermann RJ, et al: SPECT imaging of moyamoya disease using 99mTc-HM-PAO. Comparison with computed tomography findings. J Comput Tomogr. 1988, 12:247-250.

64. Nakagawara J, Takeda R, Suematsu K, et al.: Quantification of regional cerebral blood flow and vascular reserve in childhood moyamoya disease using [123I]IMP-ARG method. Clin Neurol Neurosurg. 1997, 99:96-99.

65. Ogawa A, Yoshimoto T, Suzuki J, et al: Cerebral blood flow in moyamoya disease. Part 1: Correlation with age and regional distribution. Acta Neurochir (Wien). 1990, 105:30-34.

66. Ohashi K, Fernandez-Ulloa M, Hall LC: SPECT, magnetic resonance and angiographic features in a moyamoya patient before and after external-to-internal carotid artery bypass. J Nucl Med. 1992, 33:1692-1695.

67. Rim NJ, Kim HS, Shin YS, et al: Which CT perfusion parameter best reflects cerebrovascular reserve?: correlation of acetazolamide-challenged CT perfusion with single-photon emission CT in Moyamoya patients. AJNR Am J Neuroradiol. 2008, 29:1658-1663. 10.3174/ajnr.A1229

68. Sakamoto S, Ohba S, Shibukawa M, et al: CT perfusion imaging for childhood moyamoya disease before and after surgical revascularization. Acta Neurochir (Wien). 2006, 148:77-81.

69. Morita K, Ono S, Fukunaga M, et al: [The usefulness of single photon emission computed tomography using N-isopropyl-p-[123I]-iodoamphetamine and 99mTc-

hexamethylpropyleneamine oxime in patients with moyamoya disease]. Kaku Igaku. 1990, 27:69-80.

70. Kodama N, Aoki Y, Hiraga H, et al: Electroencephalographic findings in children with moyamoya disease. Arch Neurol. 1979, 36:16-19.

71. Imaizumi T, Hayashi K, Saito K, et al: Long-term outcomes of pediatric moyamoya disease monitored to adulthood. Pediatr Neurol. 1998, 18:321-325.

72. Kurokawa T, Tomita S, Ueda K, et al: Prognosis of occlusive disease of the circle of Willis 
(moyamoya disease) in children. Pediatr Neurol. 1985, 1:274-277.

73. Ezura M, Yoshimoto T, Fujiwara S, et al: Clinical and angiographic follow-up of childhoodonset moyamoya disease. Childs Nerv Syst. 1995, 11:591-594.

74. Kuroda S, Ishikawa T, Houkin K, et al: Incidence and clinical features of disease progression in adult moyamoya disease. Stroke. 2005, 36:2148-2153.

75. Kobayashi E, Saeki N, Oishi H, et al: Long-term natural history of hemorrhagic moyamoya disease in 42 patients. J Neurosurg. 2000, 93:976-980.

76. Yoshida Y, Yoshimoto T, Shirane R, et al: Clinical course, surgical management, and longterm outcome of moyamoya patients with rebleeding after an episode of intracerebral hemorrhage: An extensive follow-Up study. Stroke. 1999, 30:2272-2276.

77. Kawaguchi S, Okuno S, Sakaki T: Effect of direct arterial bypass on the prevention of future stroke in patients with the hemorrhagic variety of moyamoya disease. J Neurosurg. 2000, 93:397-401.

78. Yasargil MG: Diagnosis and indications for operations in cerebrovascular occlusive diseases . Microsurgery Applied to Neurosurgery. Yasargil MG (ed): Georg-Thieme-Verlag, Stuttgart; 1969. 95-105.

79. Krayenbuhl HA: The Moyamoya syndrome and the neurosurgeon. Surg Neurol. 1975, 4:353360.

80. Karasawa J, Kikuchi H, Furuse S, et al: A surgical treatment of "moyamoya" disease "encephalo-myo synangiosis". Neurol Med Chir (Tokyo). 1977, 17:29-37.

81. Matsushima T, Fujiwara S, Nagata S, et al: Surgical treatment for paediatric patients with moyamoya disease by indirect revascularization procedures (EDAS, EMS, EMAS). Acta Neurochir (Wien). 1989, 98:135-140.

82. Matsushima Y, Fukai N, Tanaka K, et al: A new surgical treatment of moyamoya disease in children: a preliminary report. Surg Neurol. 1981, 15:313-320.

83. Caldarelli M, Di Rocco C, Gaglini P: Surgical treatment of moyamoya disease in pediatric age. J Neurosurg Sci. 2001, 45:83-91.

84. Dauser RC, Tuite GF, McCluggage CW: Dural inversion procedure for moyamoya disease. Technical note. J Neurosurg. 1997, 86:719-723.

85. Escanero-Salazar A, Paredes-Diaz E, Lazareff JA, et al: [Encephalo-dura-arterial synanastomosis in children with moyamoya disease]. Bol Med Hosp Infant Mex. 1990, 47:795.

86. Estridge MN: Surgical treatment of moyamoya disease: operative technique for encephalodura-arterio-myosangiosis.. Neurosurgery. 1993, 33:770-771.

87. Hanggi D, Mehrkens JH, Schmid-Elsaesser R, et al.: Results of direct and indirect revascularisation for adult European patients with Moyamoya angiopathy. Acta Neurochir. 2008, 103:119-122.

88. Houkin K, Kuroda S, Nakayama N: Cerebral revascularization for moyamoya disease in children. Neurosurg Clin N Am. 2001, 120:575-584.

89. Scott RM: Dural inversion for moyamoya disease. J Neurosurg. 1998, 88:177-178.

90. Vilela MD, Newell DW: Superficial temporal artery to middle cerebral artery bypass: past, present, and future. Neurosurg Focus. 2008, 24:10.3171/FOC/2008/24/2/E2

91. Newell DW, Dailey AT, Skirboll SL: Intracranial vascular anastomosis using the microanastomotic system. Technical note. J Neurosurg. 1998, 89:676-681.

92. Reis CV, Safavi-Abbasi S, Zabramski JM, et al: The history of neurosurgical procedures for moyamoya disease. Neurosurg Focus. 2006, 20:

93. Kohno K, Oka Y, Kohno S, et al: Cerebral blood flow measurement as an indicator for an indirect revascularization procedure for adult patients with moyamoya disease. Neurosurgery. 1998, 42:752-757.

94. Liu HM, Peng SS, Li YW: The preoperative and postoperative cerebral blood flow and vasoreactivity in childhood moyamoya disease. Keio J Med. 2000, 49:86-89.

95. Matsushima T, Inoue T, Suzuki SO, et al: Surgical treatment of moyamoya disease in pediatric patients-comparison between the results of indirect and direct revascularization procedures. Neurosurgery. 1992, 31:401-405.

96. Miyamoto S, Akiyama Y, Nagata I, et al: Long-term outcome after STA-MCA anastomosis for moyamoya disease. Neurosurg Focus. 1998, 5:

97. Morimoto M, Iwama T, Hashimoto N, et al: Efficacy of direct revascularization in adult Moyamoya disease: haemodynamic evaluation by positron emission tomography. Acta Neurochir (Wien). 1999, 141:377-384. 
Cureus

98. Nakashima H, Meguro T, Kawada S, et al.: Long-term results of surgically treated moyamoya disease. Clin Neurol Neurosurg. 1997, 99:156-161.

99. Okada Y, Shima T, Nishida M, et al: of superficial temporal artery- middle cerebral artery anastomosis in adult moyamoya disease: cerebral hemodynamics and clinical course in ischemic and hemorrhagic varieties. Stroke. 1998, 29:625-630. 